\title{
Visualisations dans l'eau pour l'étude d'écoulements turbulents
}

\section{Visualization in water for studying turbulent flows}

\section{P. Fraunié}

Institut de mécanique statistique de la turbulence

Marseille

Une revue des techniques de visualisation des écoulements turbulents est présentée sous forme de confrontation avec des résultats de calcul pour lesquels elles apportent des informations qualitatives précieuses. Certaines techniques permettent d'aborder des problèmes aux conditions aux limites complexes ou instationnaires et un développement récent des méthodes de traitement de l'image apporte une dimension quantitative aux résultats obtenus.

A review of the visualization thechniques for turbulent flows is described in confrontation form, together with calculation results providing vital qualitative information. Some techniques enable problems with complex or unsteady boundary conditions to be approached, and a recent development concerning image processing methods brings a quantitative dimension to the results obtained.

\section{Introduction}

La visualisation apporte des informations qualitatives précieuses pour l'analyse phénoménologique d'écoulements turbulents, concernant notamment la mise en évidence de structures et les mécanismes de diffusion turbulente. Celle-ci caractérise les régions turbulentes et les échanges dans les régions d'intermittence. Ces informations deviennent quantitatives par mesure de contours de fronts ou de vitesse de déplacement du traceur par traitement numérique d'acquisition d'images.

Nous nous intéresserons ici aux techniques de visualisation dans l'eau par émission de filets colorés ou de rideaux de bulle d'hydrogène. Ces techniques sont cou- ramment utilisées dans différents laboratoires pour l'étude d'écoulements cisaillés.

Lorsque l'installation le permet, la visualisation dans l'eau peut être directement associée à des mesures quantitatives par vélocimétrie laser dont le traitement du signal permet de retrouver les structures périodiques. La visualisation permet alors de décrire les mécanismes de formation et d'évolution de ces structures et la répartition spectrale observée.

Nous présenterons quelques exemples de visualisations d'écoulements turbulents à conditions aux limites instationnaires. Dans ce dernier cas, des comparaisons quantitatives peuvent être effectuées sur la géométrie des lignes d'émission et la cinématique des structures tourbillonnaires observées avec les résultats de calculs menés en repère Lagrangien. 


\section{Dispositifs expérimentaux}

La technique de visualisation peut être utilisee en canal hydrodynamique dans lequel on placera une maquette dans un écoulement uniforme ou une maquette en mouvement dans l'eau au repos. On cherchera à reproduire des expériences réalisées en soufflerie aérodynamique. Cependant les conditions de similitude sont difficiles à satisfaire, notamment sur le nombre de Reynolds limité par les faibles vitesses obtenues dans l'eau. Notons que les techniques de visualisation dans l'air par fumées ont connu un grand développement depuis l'avènement des plans laser très fins permettant de limiter l'intégration spaciale (tomographie). Les phénomènes tridimensionnels sont alors caractérisés par des discontinuités de lignes d'émission. Cependant, la visualisation en canal hydrodynamique présente encore des facilités de mise en œuvre: le suivi des particules émises (colorant ou bulles), notamment par acquisition par une caméra matricielle, est plus aisé à basses vitesses et il est possible d'étudier les régions d'interaction par l'emploi de plusieurs couleurs. De plus, des mesures quantitatives du champ de vitesse sont possibles par chronophotographie par éclairs de différentes couleurs d'un filet blanc ou d'un front d'émission séquentielle de bulles. Certaines précautions doivent être prises afin que le traceur soit un contaminant passif de l'écoulement: les propriétés physiques de celui-ci seront aussi voisines que possible de celles de l'eau. Ainsi, la densité d'un colorant pourra être ajustée par solution saline. Dans l'étude des écoulements turbulents, la visualisation apporte des informations capitales sur les structures cohérentes qui sont anisotropes et non homogènes et ne sont pas accessibles aux modèles de turbulence généralement employés. D’un point de vue expérimental, une attention particulière sera apportée à la représentation dans le plan spectral des phénomènes observés : la contribution en haute fréquence dépendra du nombre de Schmidt, laminaire ou turbulent, caractérisant la diffusivité du colorant et les fronts de structures apportent une contribution en haute fréquence à distinguer des microéchelles observables. Rappelons que dans l'eau, les échelles de Kholmogorov sont de l'ordre du dixième de millimètre et sont donc observables de visu. Le tunnel de visualisation de l'I.M.S.T. a été conçu sur un modèle classique [1] mais fonctionne de façon continue par gravité à niveau constant, ce qui permet d'étudier la stabilité d'un écoulement établi et de mener des expériences à conditions aux limites instationnaires. De plus, il est alors possible d'effectuer des campagnes de mesures quantitatives par vélocimétrie laser-doppler directement sur les expériences visualisées. Notons que dans l'eau, l'ensemencement naturel est généralement suffisant et que les échelles de turbulence rencontrées sont généralement compatibles avec la réponse en fréquence d'un suiveur.

\section{Quelques exemples de visualisation}

Nous nous limiterons ici à quelques exemples récents qui ont été comparés à des calculs. Une revue plus générale des études menées sur ce sujet sera trouvée [2].
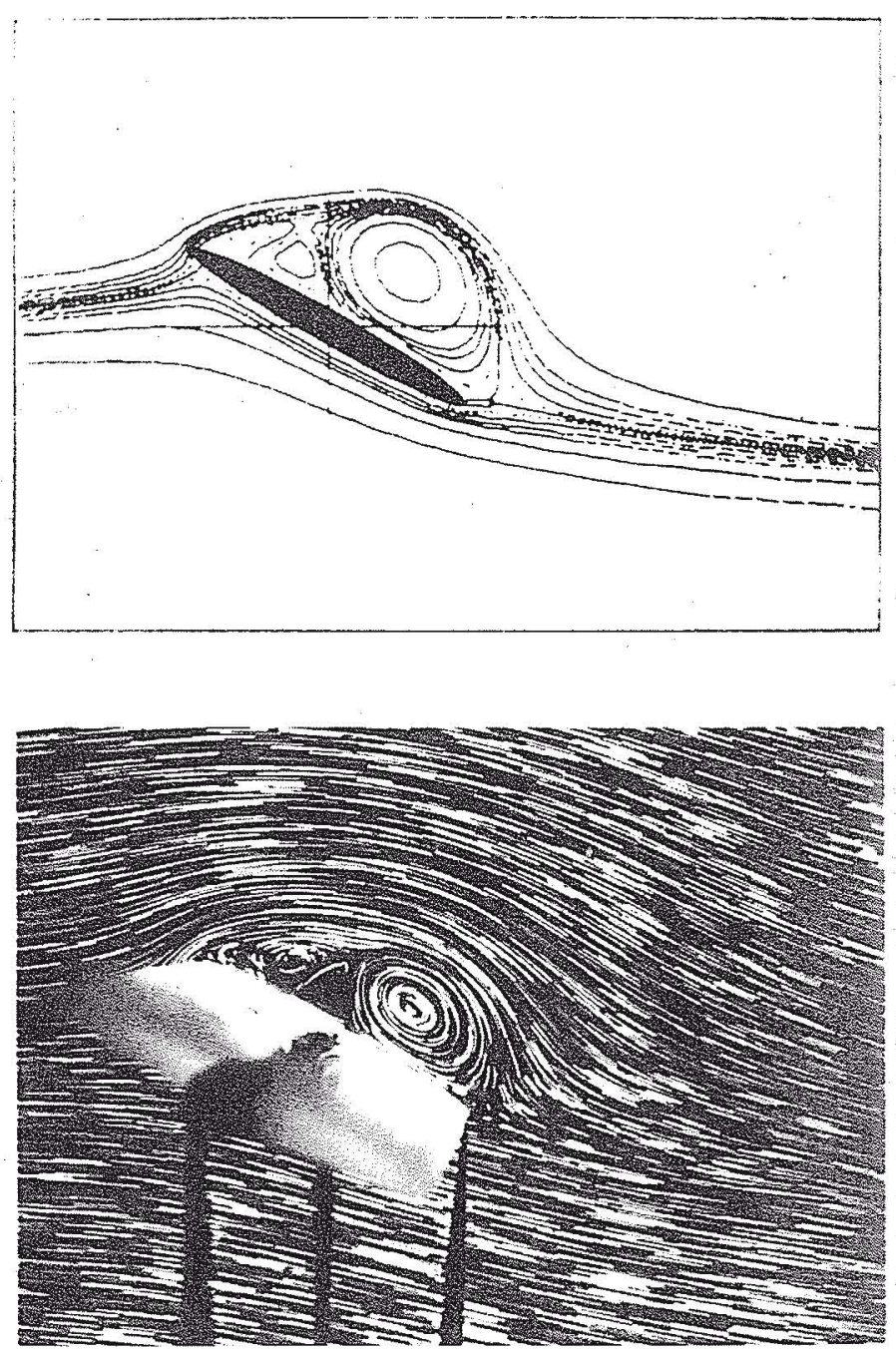

1. Décollement d'un profil elliptique en démarrage impulsif pour un Rejnolds de 1000. Visualisation et calcul (L.M.F. Poitiers L.I.M.S.I.)

L'écoulement à faible nombre de Reynolds autour d'un profil d'aile en régime décollé visualisé par particules solides a été comparé au calcul par résolution des équations de Navier-Stokes selon une méthode hermitienne compacte [3]. On comparera sur la figure $I$ la représentation des structures obtenues et l'évolution de la composante axiale de vitesse sur une normale au profil, obtenue par mesure de la trace de particules.

Des représentations de sillages tourbillonnaires peuvent être obtenues par calcul en fluide parfait instationnaire: la figure 2 représente l'émission tourbillonnaire d'aubes en rotation [4] [5] comparée à des moyennes de phase de mesure de pression mesurées en soufflerie aérodynamique. La figure 3 compare la visualisation par filets colorés de l'écoulement décollé en aval d'un profil d'aile en rotation. La comparaison des concepts de représentation en repère Lagrangien du sillage par nappes continues [6] ou particules tourbillonnaires [7] montre les limitations de ces modèles non visqueux dans les régions d'enroulement. De plus, la structure du champ de vitesse observé peut être comparée aux mesures des champs 

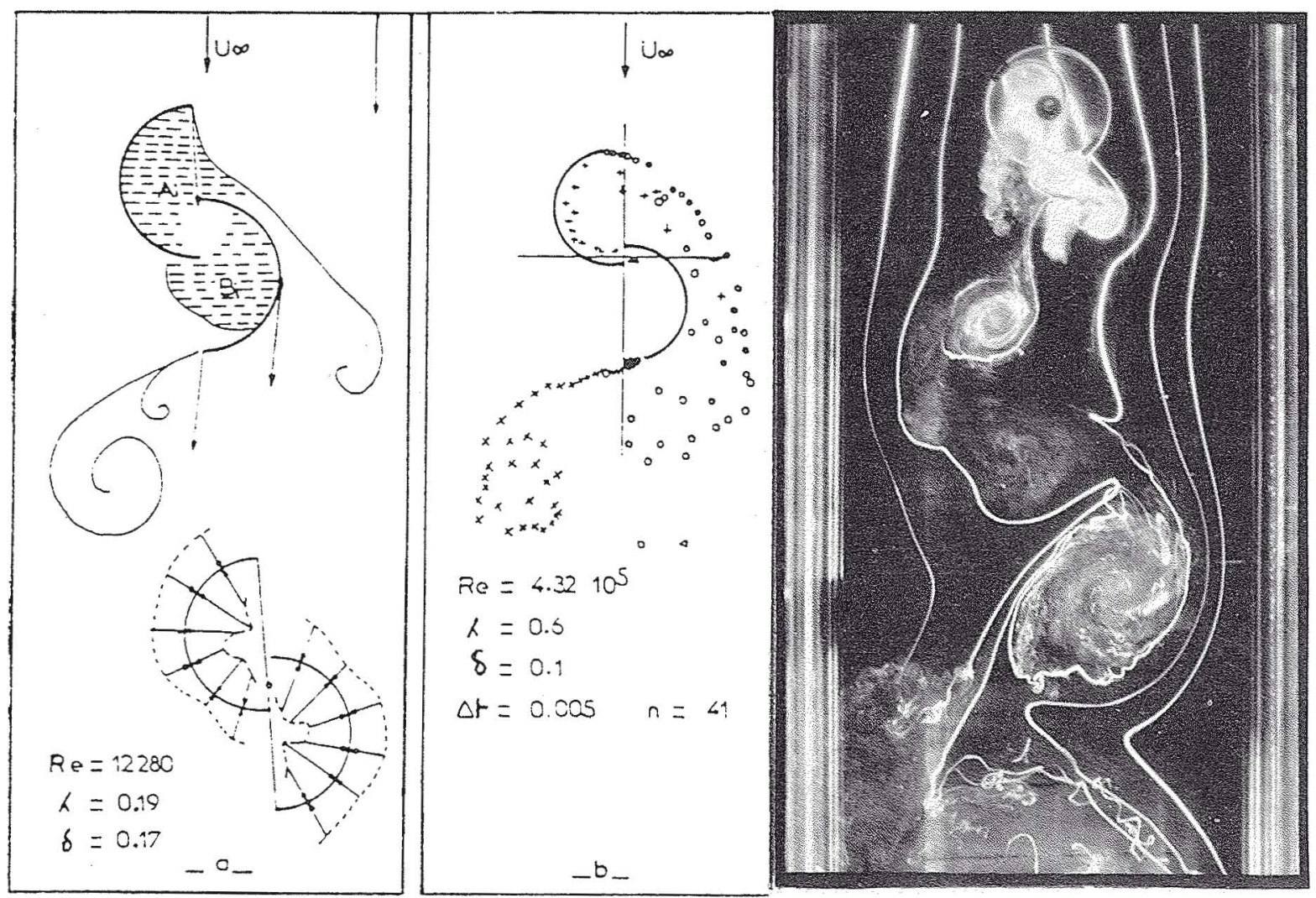

2. Emission tourbillomaire en aval d'aubes Saromius.

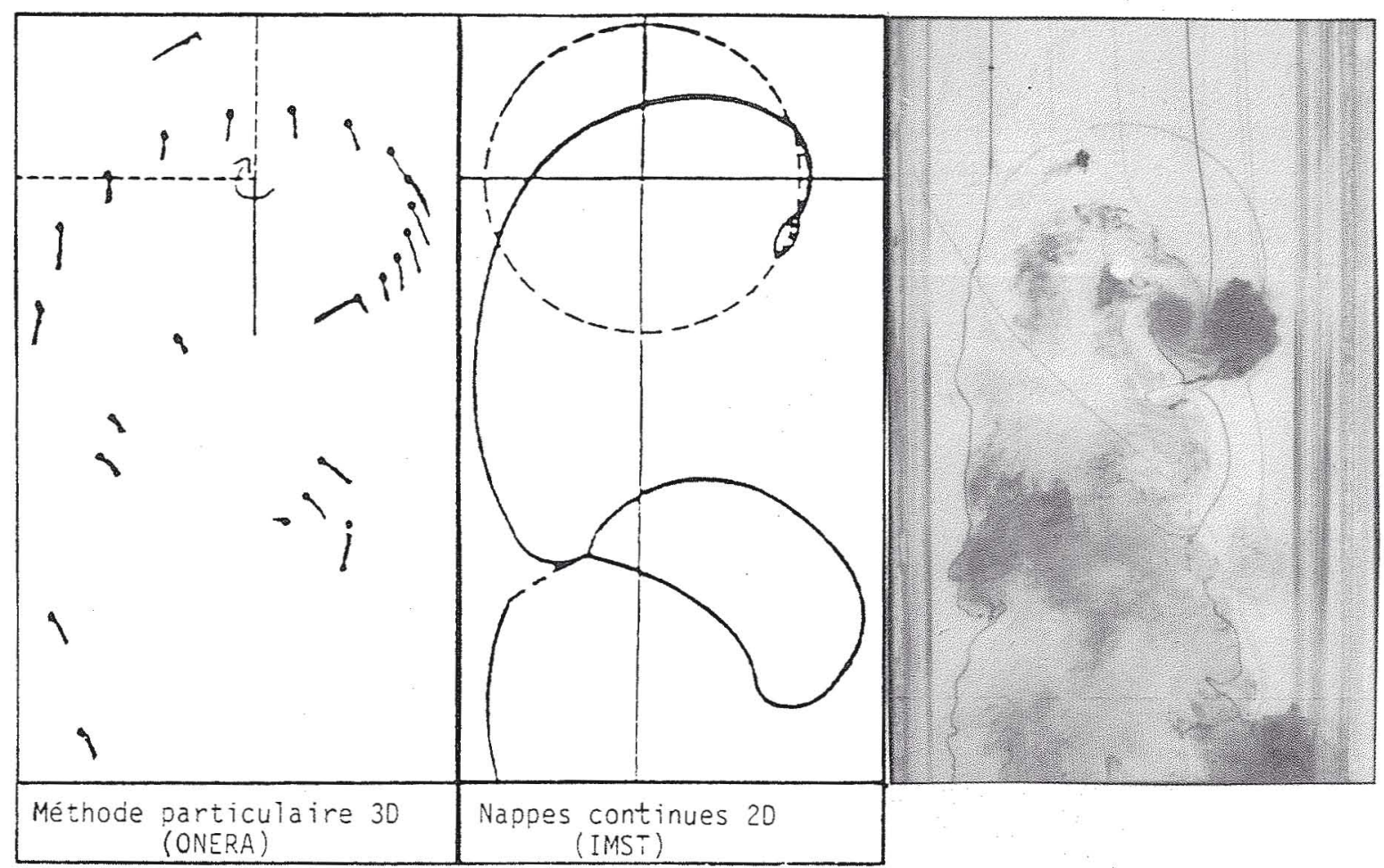

3. Sillage tourbillonnaire d'un profil d'aile en rotation.'Visualisation, calculs en fluide parfait et champ de vitesse (I.M.S.T. ONERA). 

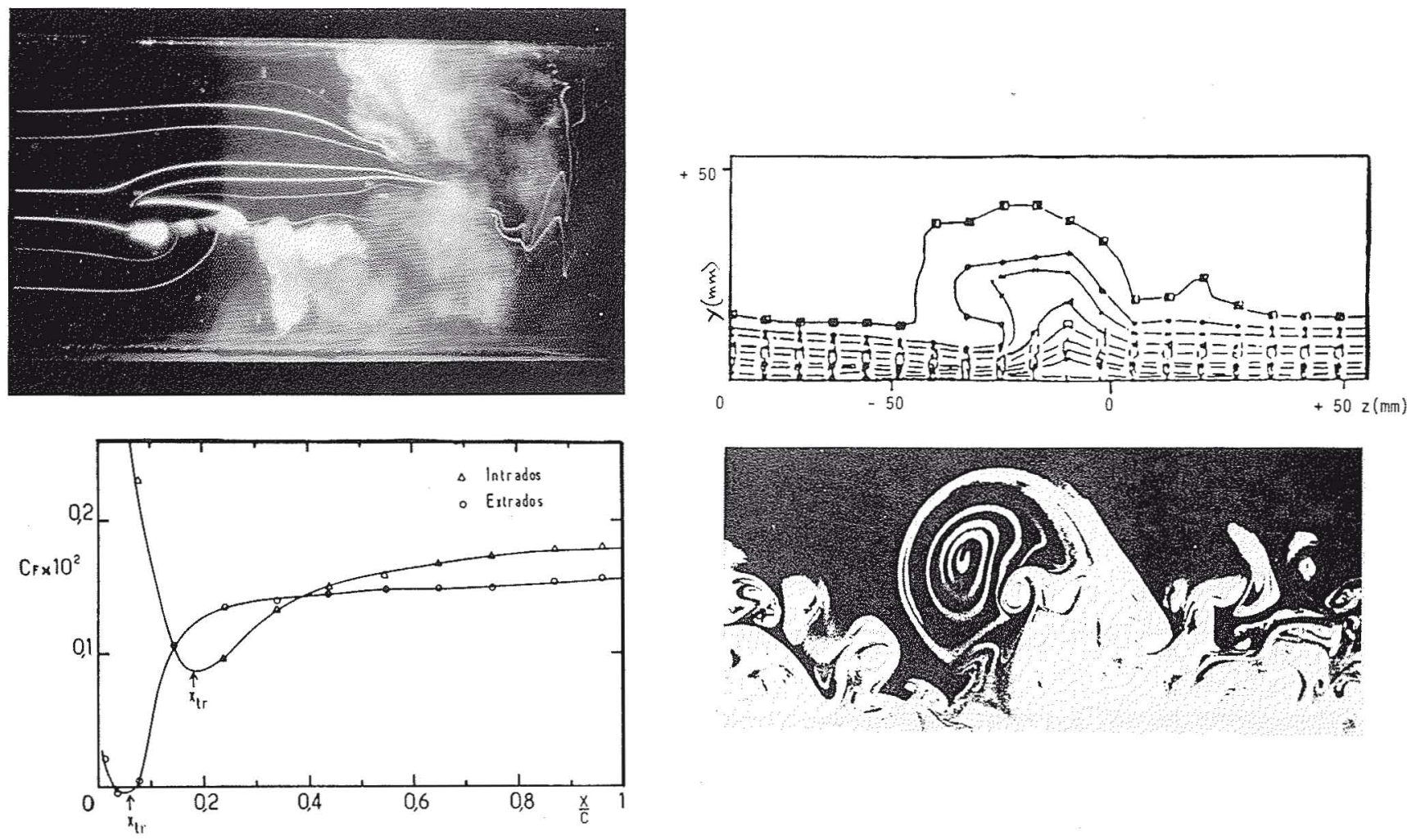

4. Couche limite sur un profil d'aile en rotation. Visualisation et calcul du coefficient de frottement pariétal (I.M.S.T.).

5. Interaction d'une structure tourbillonnaire avec une couche limite-turbulente. Visualisation et calcul (E.C.L. et ONERACERT).
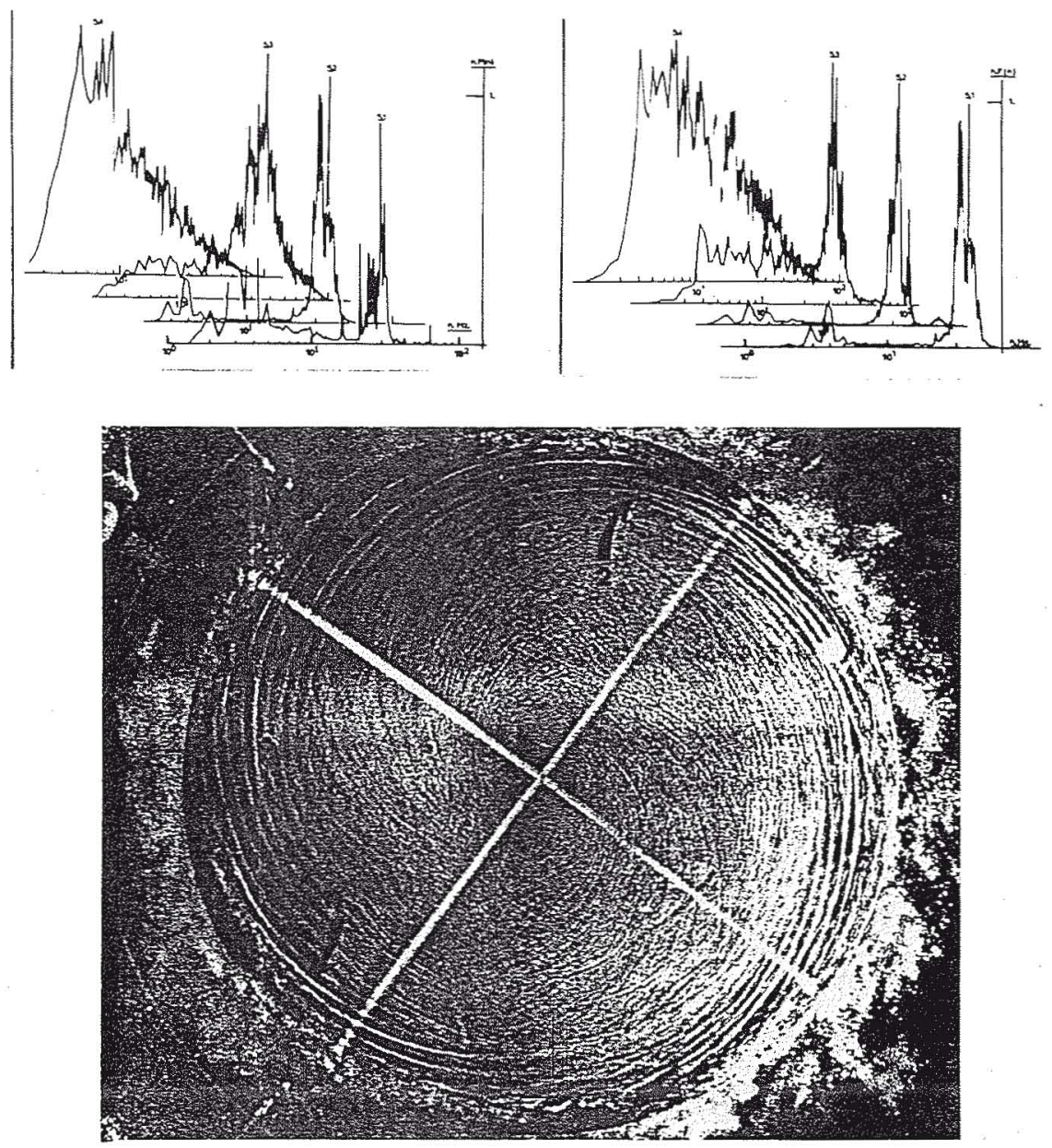

6. Instabilités sur disque tournant. Visualisation et mesures spectrales (I.M.S.T.). 
moyens et turbulents par vélocimétrie laser. On distinguera dans le sillage une région dominée par les allées tourbillonnaires alternées en aval du profil remontant l'écoulement à faible incidence, une région médiane de diffusion importante et une région caractérisée par le passage périodique de structures tourbillonnaires contrarotatives issues du décollement de l'écoulement autour de la pale descendante. Ces structures sont précisément retrouvées par l'analyse spectrale des fluctuations de vitesse. D'autre part, la structure de la couche limite laminaire et turbulente est comparée (figure 4) entre visualisation et calcul par résolution des équations de Prandtl instationnaires avec un modele de fermeture de type longueur de mélange. L'apparition d'un bulbe de décollement laminaire est caractérisée par une valeur négative de coefficient de frottement. La transition immédiate provoque le recollement de la couche limite turbulente. L'interaction d'une structure tourbillonnaire et d'une couche limite turbulente a été étudiée expérimentalement dans l'air par visualisations laser et anémométrie à fil chaud [2]. Une configuration analogue a été étudiée numériquement [8] à partir des équations de Navier-Stokes tridimensionnelles et de modèles de fermeture en un point avec équations de transport pour les tensions de Reynolds. La figure 5 présente une comparaison qualitative de deux études.

Enfin, une expérience concernant l'apparition d'instabilités sur un disque tournant dans l'eau [9] est présentée (figure 6) où les instabilités de Stuart sont caractérisées par visualisation par dépôt de colorant et par anémométrie à fil chaud.

Les visualisations permettent donc de critiquer certaines hypothèses de calcul, de valider des résultats concernant les lignes d'émission et les champs de vitesse, mais aussi de caractériser des phénomènes physiques tels que l'apparition de structures cohérentes et régions d'intermittence dont la définition théorique n'est pas établie.

\section{Conclusion}

Les techniques de visualisation, puis de traitement de l'image se sont considérablement développées et diversifiées dans des domaines d'application très variés (fluides, thermographie, météorologie par satellites, etc.). Tout d'abord qualitatives, elles ont permis d'illustrer et de comprendre des mécanismes fins, notamment dans l'étude des écoulements turbulents. Par trajectographie et chronophotographie, elles ont rapidement apporté des informations quantitatives complémentaires des méthodes d'investigation expérimentale classiques. Elles nécessitent dorénavant des moyens sophistiqués tels que des caméras à grande vitesse et des places mémoire suffisantes pour l'acquisition d'images.

Il est habituel de visualiser un phénomène physique dont on recherche une modélisation, le calcul étant alors appliqué aux conditions expérimentales choisies. Une nouvelle démarche apparaît, qui consiste à monter des expériences préliminaires de visualisation dans le but de retrouver des phénomènes observés lors d' «expériences numériques ». La relative simplicité de mise en œuvre, la richesse des informations qu'elles apportent et les conclusions que l'on peut rapidement en tirer ouvrent aux visualisations un avenir prometteur dans l'étude des nouveaux modèles théoriques d'approche des écoulements turbulents.

Remerciements: L'auteur remercie M. R. Dumas pour ses conseils lors de la préparation de cet article.

\section{Références}

[1] Werlé H. - "Le tunnel hydrodynamique au service de la recherche aérospatiale », Publication ONERA, 156, 1974.

[2] Colloque national de visualisation et de trattement d'images, A.N.R.T. - L.E.M.T.A., Nancy, 15-17 janvier 1985.

[3] Daube O., Loc T.P., Coutanceau M., Monnet P.. - Int. Conf. of Computational Methods and Experimental Measurements, Washington, July 1982.

[4] Botrini M., Beguier C., Chauvin A., Brun R. - C.R. Acad. Sc. Paris, T 298, Série II, n 20, 1984

[5] Ogawa T. - J. of Fluids Engineering, March, Vol. 106/85, 1984.

[6] Fraunie P., Brochier G., Beguier C., Paraschivolu I., European Wind Energy Conference, Rome, 7-9 octobre 1986.

[7] RehbaCh C. - A.I.A.A. I6th Aerospace Sc. Meeting, Alabama, janvier 16-18, 1978.

[8] Liandrat J., Aupolx B., Cousteix J. - 5th Symposium on Turbulent Shear Flow', Cornell University, Ithaca, USA, 1985.

[9] Chauve M.P., Tavera G. - Rencontre sur les « Structures cellulaires dans les Instabilités - Périodicité, Défauts, Turbulence de Phase ", Gif-sur-Yvette, 20-28 juin 1983, Lectures Notes in Physics $\mathrm{n}^{\circ} 210$, Springer-Verlag.

\section{Copyright}

Les figures 1, 2, 3, 4 et 5 sont des originaux I.M.S.T. non publiés. La figure 6 est extraite de : Lectures Notes in Physics $n^{\circ} 210$ de juin 1983, Springer-Verlag, Berlin, Heidelberg, 1984, Germany.

\author{
Adresse de l'auteur \\ Monsieur P. Fraunié \\ Institut de mécanique statistique de la turbulence, \\ U.M. 380033, Université d'Aix-Marseille II, \\ Centre National de la Recherche Scientifique, \\ 12 Av. du Général Leclerc, 13003 Marseille.
}




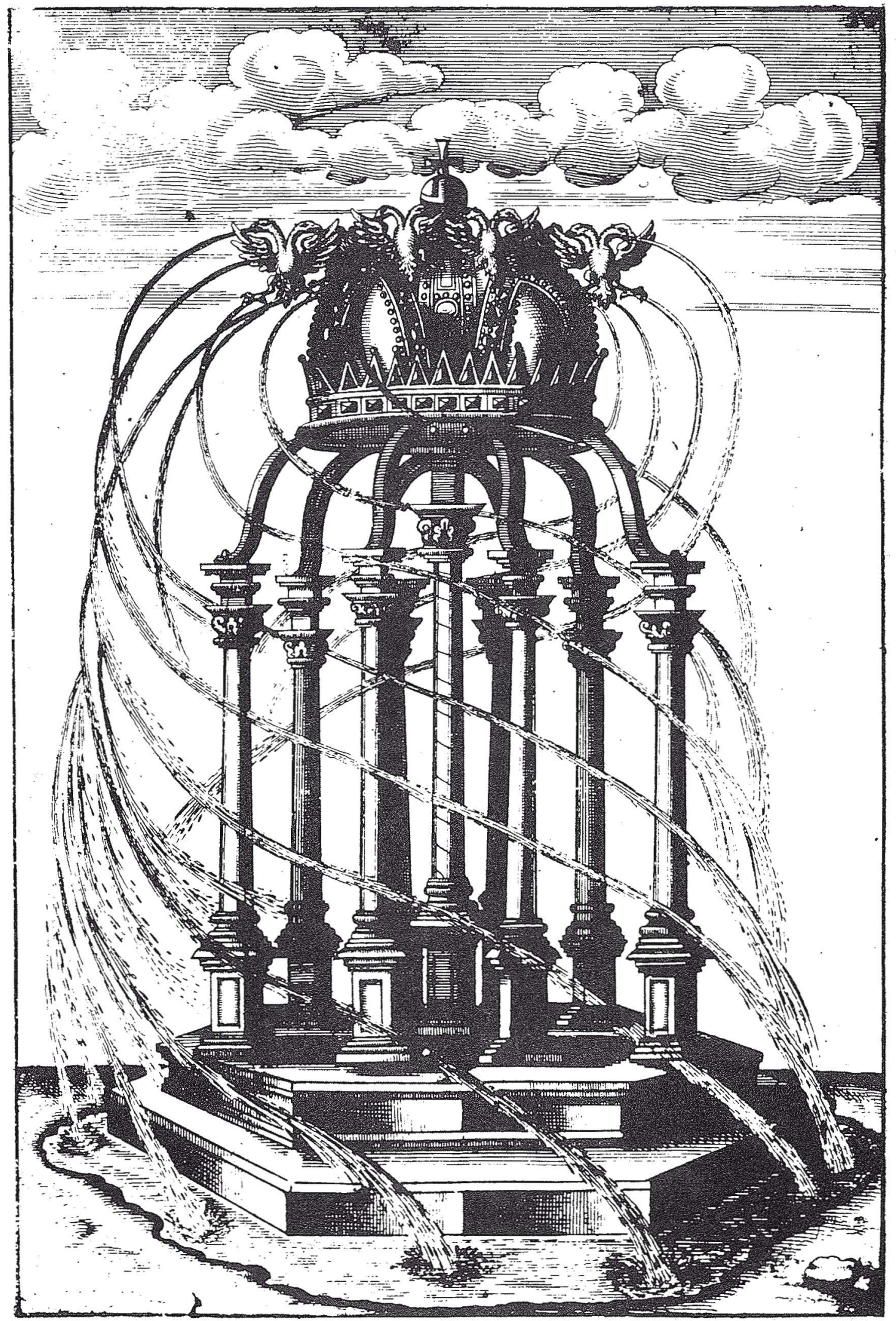

